\section{ORIGINAL RESEARCH}

J.F.A. Jansen

H.E. Stambuk

J.A. Koutcher

A. Shukla-Dave

\title{
Non-Gaussian Analysis of Diffusion-Weighted MR Imaging in Head and Neck Squamous Cell Carcinoma: A Feasibility Study
}

BACKGROUND AND PURPOSE: Water in biological structures often displays non-Gaussian diffusion behavior. The objective of this study was to test the feasibility of non-Gaussian fitting by using the kurtosis model of the signal intensity decay curves obtained from DWI by using an extended range of b-values in studies of phantoms and HNSCC.

MATERIALS AND METHODS: Seventeen patients with HNSCC underwent DWI by using 6 b-factors (0, $50-1500 \mathrm{~s} / \mathrm{mm}^{2}$ ) at $1.5 \mathrm{~T}$. Monoexponential (yielding $A D C_{\text {mono }}$ ) and non-Gaussian kurtosis (yielding apparent diffusion coefficient $D_{\text {app }}$ and apparent kurtosis coefficient $K_{\text {app }}$ ) fits were performed on a voxel-by-voxel basis in selected regions of interest (primary tumors, metastatic lymph nodes, and spinal cord). DWI studies were also performed on phantoms containing either water or homogenized asparagus. To determine whether the kurtosis model provided a significantly better fit than did the monoexponential model, an $F$ test was performed. Spearman correlation coefficients were calculated to assess correlations between $\mathrm{K}_{\mathrm{app}}$ and $\mathrm{D}_{\mathrm{app}}$

RESULTS: The kurtosis model fit the experimental data points significantly better than did the monoexponential model $(P<.05)$. $\mathrm{D}_{\text {app }}$ was approximately twice the value of $A D C_{\text {mono }}$ (eg, in neck nodal metastases $D_{\text {app }}$ was 1.54 and $A D C_{\text {mono }}$ was 0.84$)$. $K_{\text {app }}$ showed a weak Spearman correlation with $D_{a p p}$ in a homogenized asparagus phantom and for $44 \%$ of tumor lesions.

CONCLUSIONS: The use of kurtosis modeling to fit DWI data acquired by using an extended b-value range in HNSCC is feasible and yields a significantly better fit of the data than does monoexponential modeling. It also provides an additional parameter, $K_{a p p}$ potentially with added value.

ABBREVIATIONS: $A D C=$ apparent diffusion coefficient; $B O T=$ base of tongue; $D W I=$ diffusionweighted imaging; Gd-DTPA = gadolinium-diethylene-triamine pentaacetic acid; $\mathrm{HN}=$ head and neck; HNSCC = head and neck squamous cell carcinoma; NPC = nasopharyngeal cancer; $\mathrm{ROI}=$ region of interest; $\mathrm{SCC}=$ squamous cell carcinoma; SNR = signal intensity-to-noise ratio

$T^{T}$ he MR imaging technique known as DWI allows measurement of water diffusivity. ${ }^{1}$ Because freedom of translational motion of water molecules is hindered by interactions with other molecules and cell membranes, DWI abnormalities can reflect changes of tissue organization at the cellular level. ${ }^{2,3}$ These microstructural changes affect the motion of water molecules, and consequently alter the water diffusion properties and thus the MR imaging signal intensity. The signal intensity loss in DWI can be quantified by using the ADC, which is a measure of the average molecular motion that is affected by cellular organization and integrity. In the simplest models, the distribution of a water molecule diffusing from one location to another in a certain period of time is considered to have a Gaussian form with its width proportional to the ADC. ${ }^{2}$ How-

Received June 10, 2009; accepted after revision September 7

From the Departments of Medical Physics (J.F.A.J., J.A.K., A.S.-D.), Radiology (J.F.A.J., H.E.S., J.A.K., A.S.-D.), and Medicine (J.A.K.), Memorial Sloan-Kettering Cancer Center, New York, New York.

This work was supported by National Cancer Institute/National Institutes of Health (Grant 1 R01 CA115895).

Previously presented in part as abstract 1319 at: 17th Annual Meeting of the International Society of Magnetic Resonance in Medicine, April, 18-24, 2009, Honolulu, Hawaii.

Please address correspondence to Amita Shukla-Dave, PhD, Department of Medical Physics and Radiology, Memorial Sloan-Kettering Cancer Center, 1275 York Ave, New York, NY 10021; e-mail: davea@mskcc.org

Indicates open access to non-subscribers at www.ajnr.org

DOI 10.3174/ajnr.A1919 ever, water in biological structures often displays non-Gaussian diffusion behavior. ${ }^{4}$ As a result, the MR signal intensity decay in tissue is not a simple monoexponential function of the b-value. ${ }^{3,5}$

Several approaches have been used to model the nonlinear decay of DWI signal intensity when more than $2 \mathrm{~b}$-values are acquired. These approaches include biexponential fitting, from which 2 components that hypothetically reflect 2 separate biophysical compartments can be derived, ${ }^{6}$ stretchedexponential fitting, which describes diffusion-related signal intensity decay as a continuous distribution of sources decaying at different rates, ${ }^{7}$ and diffusional kurtosis analysis, which takes into account non-Gaussian properties of water diffusion by measuring the kurtosis. ${ }^{8}$ Kurtosis represents the extent to which the diffusion pattern of the water molecules deviates from a perfect Gaussian curve. Unlike the biexponential model, the stretched-exponential and the kurtosis methods do not make assumptions regarding the number of biophysical compartments or even the existence of multiple compartments. ${ }^{3}$ From the kurtosis analysis, 2 parameters are derived: the apparent diffusion coefficient $\left(\mathrm{D}_{\mathrm{app}}\right)$ and the apparent kurtosis coefficient $\left(\mathrm{K}_{\mathrm{app}}\right)$.

In the $\mathrm{HN}$ region, DWI has been used for characterizing and differentiating benign and malignant pathology in patients, ${ }^{9-19}$ evaluating treatment-induced tissue changes, especially after chemoradiation therapy, ${ }^{20-22}$ and assessing persistent or recurrent cancer. ${ }^{23,24}$ In previous DWI studies in HN 


\begin{tabular}{lcll}
\hline \multicolumn{4}{l}{ Table 1: Patient characteristics } \\
\hline Subject & Age (y)/Sex & $\begin{array}{l}\text { Primary } \\
\text { Cancer }\end{array}$ & \multicolumn{1}{c}{ ROls } \\
\hline 1 & $65 / \mathrm{M}$ & NPC & Primary, 2 nodes, and spinal cord \\
2 & $75 / \mathrm{F}$ & Tonsil & Primary, node, and spinal cord \\
3 & $84 / \mathrm{M}$ & BOT & Primary and spinal cord \\
4 & $55 / \mathrm{M}$ & BOT & Primary, 2 nodes, and spinal cord \\
5 & $70 / \mathrm{M}$ & Tonsil & Primary, node, and spinal cord \\
6 & $78 / \mathrm{M}$ & BOT & Primary and node \\
7 & $27 / \mathrm{F}$ & Tonsil & Primary, 2 nodes, and spinal cord \\
8 & $60 / \mathrm{M}$ & Tonsil & Primary and node \\
9 & $63 / \mathrm{M}$ & BOT & Primary, node, and spinal cord \\
10 & $66 / \mathrm{M}$ & BOT & 3 nodes and spinal cord \\
11 & $51 / \mathrm{M}$ & BOT & Primary and node \\
12 & $60 / \mathrm{M}$ & BOT & Primary and 3 nodes \\
13 & $51 / \mathrm{F}$ & Tonsil & Primary, 2 nodes, and spinal cord \\
14 & $51 / \mathrm{M}$ & Tonsil & Primary, 2 nodes, and spinal cord \\
15 & $68 / \mathrm{M}$ & Tonsil & 3 nodes and spinal cord \\
16 & $55 / \mathrm{F}$ & Tonsil & Primary and node \\
17 & $49 / \mathrm{F}$ & NPC & Primary, 2 nodes, and spinal cord \\
\hline
\end{tabular}

cancer patients, a combination of 2 or 3 b-factors (eg, 0,500 , and $1000 \mathrm{~s} / \mathrm{mm}^{2}$ ) was usually acquired, and the ADC values $\left(\mathrm{ADC}_{\text {mono }}\right)$ were calculated assuming that the signal intensity decay is a monoexponential function. ${ }^{12,18,19,25}$ To our knowledge, no study has considered the non-Gaussian diffusion behavior of DWI data in HNSCC. The kurtosis model may lead to better fit of the experimental data. ${ }^{8}$ The diffusion parameters $\mathrm{D}_{\text {app }}$ and $\mathrm{K}_{\text {app }}$ provided by this model could offer potential value for longitudinal studies assessing early response in patients with HNSCC undergoing chemoradiation therapy.

The objective of this study was to test the feasibility of nonGaussian fitting by using the kurtosis model of the signal intensity decay curves obtained from DWI by using an extended range of b-values in studies of phantoms and HNSCC.

\section{Materials and Methods}

\section{Phantoms}

Phantoms were prepared containing either Gd-DTPA-doped water or homogenized asparagus. ${ }^{8}$ Gd-DTPA was obtained from Magnevist (Bayer HealthCare Pharmaceuticals, Wayne, New Jersey).

\section{Patients}

Our institutional review board approved and issued a waiver of informed consent for our retrospective study, which was compliant with the Health Insurance Portability and Accountability Act. Between June 2008 and June 2009, 17 consecutive patients with HNSCC, referred for MR imaging by physicians at our institution, underwent a pretreatment clinical MR imaging examination that included DWI. Patient characteristics are given in Table 1.

SCC pathology was confirmed by using needle biopsy at the primary $\mathrm{HN}$ tumor location at least 1 week before the MR imaging examination. Of the sites that contributed to the mean DWI parameter values for these 17 patients, primary tumors included 6 base of tongue, 7 tonsil, and 2 nasopharyngeal tumors, 28 were metastatic neck lymph nodes, and 12 were spinal cord (which served as a control region).

\section{MR Imaging Data Acquisition}

MR imaging data were acquired on a 1.5T Excite scanner (GE Healthcare, Milwaukee, Wisconsin) by using either a 4-channel or 8-channel neurovascular phased array coil for signal intensity reception and a body coil for transmission. Accuracy was assessed on both the settings (1.5T by using a 4 -channel neurovascular array coil, $1.5 \mathrm{~T}$ by using an 8 -channel neurovascular array coil) with phantoms at $25^{\circ} \mathrm{C}$.

The patient study was done on either 4-channel $(n=10)$ or 8 -channel $(n=7)$ coils and consisted of MR imaging covering the entire neck or oral cavity/tongue or nasopharynx. The standard MR acquisition parameters were as follows: rapid scout images, multiplanar (axial, coronal, and sagittal) T2-weighted, fat-suppressed, fast spin-echo images (TR, $5000 \mathrm{~ms}$; TE, $102 \mathrm{~ms}$; averages, 2; matrix, $256 \times 256$; section thickness, $5.0 \mathrm{~mm}$; and gap, $2.5 \mathrm{~mm}$ for neck survey; for oral cavity/tongue or nasopharynx imaging, section thickness, $4.0 \mathrm{~mm}$; gap, $1.0 \mathrm{~mm}$ ), multiplanar T1-weighted images (TR, $675 \mathrm{~ms}$; TE, $8 \mathrm{~ms}$; averages, 2; section thickness, $5.0 \mathrm{~mm}$; gap, $2.5 \mathrm{~mm}$; matrix, $256 \times 256$ ). Standard T1- and T2-weighted imaging was followed by DWI. After DWI, Gd-DTPA contrast agent was injected and postcontrast multiplanar T1-weighted images were acquired.

For patients, diffusion-weighted images were obtained by using single-shot spin-echo echo-planar imaging with a pair of rectangular gradient pulses along 3 orthogonal axes. The imaging parameters were as follows: TR, $4000 \mathrm{~ms}$; TE, 85-98 ms; averages, 4; section thickness, $5.0 \mathrm{~mm}$; gap, $0 \mathrm{~mm}$; FOV, $20-26 \mathrm{~cm}$; matrix, $128 \times 128$; and 3 diffusion sensitizing directions. The b-values were $0,50,100,500,750$, 1000 , and $1500 \mathrm{~s} / \mathrm{mm}^{2}$. The duration of the DWI component of the examination was approximately 8 minutes. Four to 10 sections were acquired to cover the lesion.

DWI experiments were performed on phantoms by using the same type of sequence and b-values as for the HNSCC patients. There is a difference in loading of the coil between the studies of phantoms and patients. To obtain the best SNR characteristics in the phantom study, the experiments were performed with similar MR parameters as detailed above except for averages of 16 and section thickness of $15.0 \mathrm{~mm}$.

\section{Image Analysis}

Image processing was performed by using customized software in Matlab (MathWorks, Natick, Massachusetts), based on SPM5 software routines (Wellcome Department of Cognitive Neurology, London, United Kingdom). The individual diffusion-weighted images for the 3 directions were automatically averaged on the scanner; hence, for subsequent analyses only the averaged diffusion-weighted images were included. For all of the 6 DWI images with a b-value $>0$ $\mathrm{s} / \mathrm{mm}^{2}$, a corresponding image with $b=0 \mathrm{~s} / \mathrm{mm}^{2}$ was obtained. All 6 $b=0$ images were aligned to correct for motion, by using an affine transformation in SPM5. Subsequently, all corresponding DWI images with a b-value $>0 \mathrm{~s} / \mathrm{mm}^{2}$ were transformed by using the same parameters obtained from the $b=0$ images realignment. An averaged $b=0$ image was obtained after realignment, with optimal SNR characteristics. Next, Gaussian smoothing was applied on all images with a full width at half maximum of $3 \mathrm{~mm}$, which is approximately 3 times the in-plane voxel size.

For phantom data analysis, a ROI created by using the MRIcro software $^{26}$ (www.sph.sc.edu/comd/rorden/mricro.html) was positioned within the phantom of size approximately $64 \mathrm{~mm}^{3}$. Statistics (mean and SD) were derived from the values within the ROI. Combining data from both 4 - and 8 -channel coils was proved to be feasible, and no differences in ADC values were noted.

For each patient, masks were created by using MRIcro to select a ROI for each lesion (primary tumors and nodes). ROIs were manually drawn by an experienced neuroradiologist (with $>10$ years of expe- 
rience in reading MR imaging of the head and neck) on the averaged $b=0$ image. All of the sections containing the tumor or nodal metastasis were outlined and analyzed. Fits were performed for all ROIs on a voxel-by-voxel basis with a Marquardt-Levenberg algorithm implemented in Matlab, as described previously by Lu et al. ${ }^{3}$ SNR for all ROIs for all diffusion-weighted images were calculated by dividing the maximum signal intensity within the ROI by the SD of the noise within an ROI of identical size outside the body.

The signal intensity versus b-factor from each voxel in the ROI, as extracted from DWI datasets acquired in the presence of diffusionsensitizing gradients, was fitted with both monoexponential functions and non-monoexponential decay functions of the form

$$
\ln [S(b)]=\ln [S(0)]-b \times \mathrm{D}_{\mathrm{app}}+1 / 6 b^{2} \times \mathrm{D}_{\mathrm{app}}{ }^{2} \times \mathrm{K}_{\mathrm{app}},
$$

where $S$ is the signal intensity (arbitrary units), $b$ is the b-value (s/ $\left.\mathrm{mm}^{2}\right), \mathrm{D}_{\text {app }}$ is the apparent diffusion coefficient $\left(10^{-3} \mathrm{~mm}^{2} / \mathrm{s}\right)$, and $\mathrm{K}_{\mathrm{app}}$, a dimensionless parameter, is the apparent kurtosis coefficient. The fit parameters were not assigned initial values for the curve fitting. In addition to non-Gaussian fitting, monoexponential fitting was performed by using $\mathrm{K}_{\mathrm{app}}=0$ in the equation, yielding $\mathrm{ADC}_{\text {mono }}$.

Heterogeneity of tumors was assessed by computing the SD of a normalized distribution of the voxels within each ROI. ${ }^{27}$ Toward this end, the DWI-derived parameters $\mathrm{ADC}_{\text {mono }}, \mathrm{D}_{\mathrm{app}}$, and $\mathrm{K}_{\mathrm{app}}$ were first normalized for each individual ROI to a scale of $[0,100]$, with 0 as the minimum and 100 as the maximum value. Subsequently, the SD was calculated from these normalized distributions. This procedure yielded heterogeneity measures $\mathrm{hADC}_{\text {mono }}, \mathrm{hD}_{\mathrm{app}}$, and $\mathrm{hK}_{\mathrm{app}}$.

Additionally, necrosis was assessed by the radiologist separately for the primary tumor and the neck nodal metastasis in each patient on both the pre-T2-weighted images and the post Gd-DTPA contrast T1-weighted images by means of visual inspection. Necrosis is hyperintense on T2-weighted images and hypointense on postcontrast T1weighted images. The MR imaging reading was scored on a scale of [0, $1]$, with 0 indicating no necrosis and 1 indicating necrosis (which included mild to severe necrosis).

\section{Statistical Analysis}

Statistical tests were performed in SPSS for Windows (release 15.0.1; SPSS, Chicago, Illinois).

To test the goodness of fit for the monoexponential and kurtosis models, the reduced $\chi^{2}$ was calculated on a voxel-by-voxel basis, which is the sum of the differences between observed and expected outcome values, each squared and divided by the expectation, divided by the number of degrees of freedom in the model. To determine whether the kurtosis model provided a statistically improved fit over the monoexponential model, the differences between the results of the 2 approaches were tested by using an $F$ test of the resulting sum of squares values, with $P<.05$ denoting statistical significance. In detail, the monoexponential fitting model has 5 degrees of freedom ( 7 data points minus 2 variables), and the kurtosis model has 4 degrees of freedom. In this situation, the ratio of the relative increase of sum of squares divided by the increase of degrees of freedom has to be $>F(0.05,1,4)=4.5$.

To assess whether the kurtosis analysis also yields potential additional information to the standard ADC value, in terms of $\mathrm{K}_{\mathrm{app}}$, the Spearman rank-order correlation coefficient for $\mathrm{K}_{\text {app }}$ and $\mathrm{D}_{\text {app }}$ was calculated for both phantom and patient data. A high absolute Spearman correlation coefficient $(|\rho|)$ between $\mathrm{K}_{\text {app }}$ and $\mathrm{D}_{\text {app }}$ would indicate that $\mathrm{K}_{\mathrm{app}}$ does not have additional information, whereas a weak

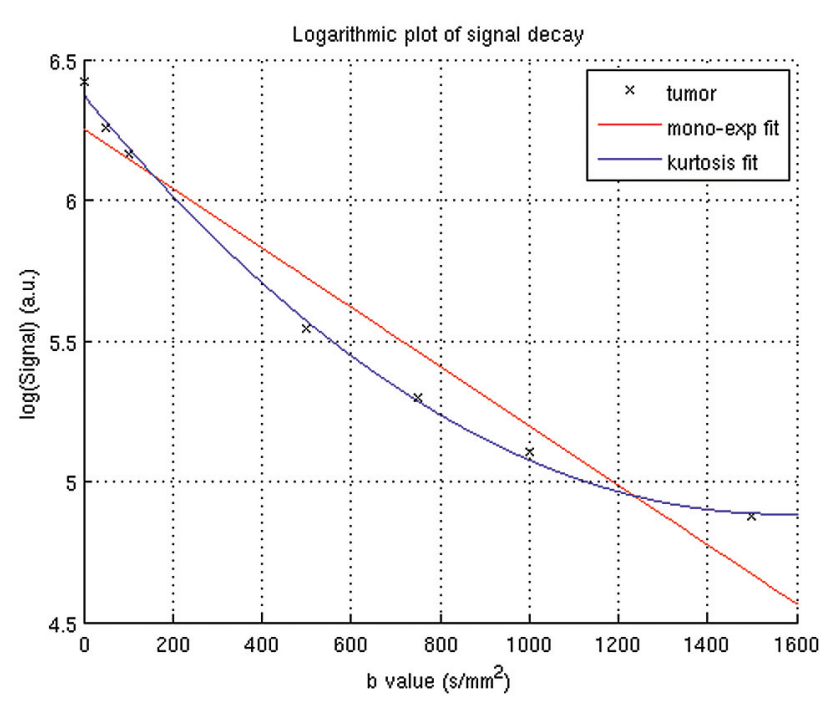

Fig 1. Logarithm of the MR signal intensity decay averaged over all voxels within the right node of patient 12 as a function of the b-value. The black crosshairs represent the experimental data, and the solid lines are the fitted curves (red is the monoexponential fit, blue is the non-Gaussian kurtosis fit).

correlation coefficient might indicate that $\mathrm{K}_{\mathrm{app}}$ is independent of $\mathrm{D}_{\text {app }}$ and therefore does reflect additional information. ${ }^{8}$

To explore the potentially increased sensitivity of $\mathrm{D}_{\text {app }}$ to reflect heterogeneity over $\mathrm{ADC}_{\text {mono }}$, the differences in heterogeneity measures $\mathrm{hADC}_{\text {mono }}$ and $\mathrm{hD}_{\text {app }}$ were statistically tested by using a 2 -sided Student $t$ test. Additionally, to investigate whether the diffusion parameters, the potential additional information of the kurtosis analysis, and the heterogeneity values of $\mathrm{ADC}_{\text {mono }}, \mathrm{D}_{\mathrm{app}}$, and $\mathrm{K}_{\mathrm{app}}$, are affected by necrosis, tumor lesions were divided into 2 categories: $0=$ no necrosis, 1 = necrosis. Subsequently, a 2 -sided Student $t$ test was applied on these measures to assess statistical differences between necrotic and non-necrotic lesions.

\section{Results}

\section{Phantom Experiments}

Phantom experiments revealed perfect Gaussian behavior for the phantom containing Gd-DTPA-doped water $\left(\mathrm{ADC}_{\mathrm{mono}}=\right.$ $2.29 \pm 0.0110^{-3} \mathrm{~mm}^{2} / \mathrm{s}, \mathrm{D}_{\text {app }}=2.31 \pm 0.0210^{-3} \mathrm{~mm}^{2} / \mathrm{s}$, and $\left.\mathrm{K}_{\mathrm{app}}=0.02 \pm 0.02\right)$ and non-Gaussian behavior for the phantom containing homogenized asparagus $\left(\mathrm{ADC}_{\text {mono }}=1.55 \pm\right.$ $0.0710^{-3} \mathrm{~mm}^{2} / \mathrm{s}, \mathrm{D}_{\mathrm{app}}=1.75 \pm 0.0810^{-3} \mathrm{~mm}^{2} / \mathrm{s}$, and $\mathrm{K}_{\mathrm{app}}=$ $0.28 \pm 0.05)$. The $\mathrm{D}_{\text {app }}$ values from the non-Gaussian kurtosis model were higher than the $\mathrm{ADC}_{\text {mono }}$ values from the monoexponential fit, as reported previously. ${ }^{3}$ The $\mathrm{K}_{\mathrm{app}}$ in the homogenized asparagus phantom was substantially higher than the $K_{a p p}$ in the water phantom, reflecting a significant departure from Gaussian diffusion. ${ }^{8}$

\section{Comparison of Fitting Methods}

Figure 1 provides an example of the signal intensity decay curve of mean intensity of the right node of patient 12 , as a function of the b-value. The plot is semilogarithmic, and the data points display a nonlinear decay. Also presented in this plot are the fitting curves from the monoexponential fitting procedure and the diffusional kurtosis analysis. It can be clearly seen that the non-Gaussian kurtosis analysis fits the data points substantially better than does the monoexponen- 
Table 2: Average estimated diffusion coefficients derived from monoexponential fitting and diffusional kurtosis analysis in patients with squamous cell carcinoma head and neck cancer $(n=17)$

\begin{tabular}{lcccc}
\hline Region $(n)$ & $\mathrm{ADC}_{\text {mono }}$ & $\mathrm{D}_{\text {app }}$ & $\mathrm{K}_{\text {app }}$ & $\begin{array}{c}\rho\left(\mathrm{D}_{\text {app }} \text { and }\right. \\
\left.\mathrm{K}_{\text {app }}\right)\end{array}$ \\
\hline Node (28) & $0.84 \pm 0.24$ & $1.54 \pm 0.45$ & $1.38 \pm 0.37$ & $-0.49 \pm 0.35$ \\
BOT primary (6) & $0.67 \pm 0.22$ & $1.32 \pm 0.35$ & $1.69 \pm 0.54$ & $-0.52 \pm 0.42$ \\
Tonsil primary (7) & $0.78 \pm 0.20$ & $1.46 \pm 0.41$ & $2.91 \pm 3.92$ & $-0.54 \pm 0.27$ \\
NPC primary (2) & $1.16 \pm 0.19$ & $2.21 \pm 0.05$ & $0.92 \pm 0.17$ & $-0.21 \pm 0.34$ \\
Spine (12) & $0.74 \pm 0.08$ & $1.44 \pm 0.29$ & $1.41 \pm 0.15$ & $-0.26 \pm 0.42$
\end{tabular}

Note: $-n$ indicates number of regions (over all squamous cell carcinoma patients) contributing to the average; $A D C_{\text {mono, }}$ apparent diffusion coefficient obtained from monoexponential fitting $\left(10^{-3} \mathrm{~mm}^{2} / \mathrm{s}\right) ; D_{a p p}$, apparent diffusion coefficient obtained from diffusional kurtosis analysis $\left(10^{-3} \mathrm{~mm}^{2} / \mathrm{s}\right): \mathrm{K}_{\text {app }}$ apparent kurtosis coefficient obtained from diffusional kurtosis analysis (dimensionless); $\rho$ (D ap and $K$ coeffic correlation coefficient for correlation between $\mathrm{K}_{\mathrm{app}}$ and $\mathrm{D}_{\text {app }}$. Averages are presented as mean \pm SD

tial fitting procedure. To assess whether these beneficial fit characteristics were also significant, an $F$ test was performed on all ROIs. The $F$ ratio obtained in this case (Fig 1) was 26.8, hence greater than $F(0.05,1,4)=4.5$, indicating that the nonGaussian kurtosis analysis was significantly $(P<.05)$ better than the monoexponential fit. For all individual cases in the whole population, the non-Gaussian kurtosis analysis was always significantly better than the monoexponential fit, as calculated from the $F$ test $(P<.05)$. The median reduced $\chi^{2}$ of all ROIs from the entire population was 0.008 (range, 0.001-0.15) for the monoexponential fit and 0.002 (range, 0.0003-0.15) for the kurtosis fit. The median SNR within the ROIs in the diffusion-weighted images acquired with the highest b-value ( $b=1500 \mathrm{~s} / \mathrm{mm}^{2}$ ) was 42 (range, $21-87$ ).

\section{Patients}

In total, 17 patients were successfully scanned by using the extended b-value protocol. Table 2 shows the average estimated diffusion coefficients for various tissue structures, obtained by using monoexponential fitting and diffusional kurtosis analysis.

The ADC obtained from the diffusional kurtosis analysis $\left(D_{\text {app }}\right)$ was about twice the value of the ADC obtained by monoexponential fitting $\left(\mathrm{ADC}_{\text {mono }}\right)$ (Table 2$)$. Figure 2 shows the MR image series obtained from the oral cavity of patient 12. In Fig $2 C-F$, the calculated fitting parameters for the right node are visualized by using a mask overlay on the realigned mean $b=0$ image. From the reduced $\chi^{2}$ error estimate in Fig $2 F$, it can be observed that the fit deteriorates near the rim of the node. In Fig $2 G-I$, histogram distribution plots based on monoexponential and non-Gaussian fitting are shown. In Fig $2 I$, the histogram for $\mathrm{K}_{\mathrm{app}}$ displays the presence of 2 distinct distribution peaks, at 0.5 and 1.1 .

\section{Potential Additional Information}

Aside from providing a better fit, the non-Gaussian kurtosis analysis may also yield new information (ie, the $\mathrm{K}_{\mathrm{app}}$ [apparent kurtosis coefficient]) in addition to the standard ADC value. To assess the potential added value of this parameter, for all voxels of the tumor highlighted in Fig $3 A$ (right node of patient 12 ), the $D_{\text {app }}$ was plotted as a function of the $K_{a p p}$. The resulting scatterplot is given in Fig 3C. To investigate this relationship in a quantitative way, a Spearman rank-order correlation coefficient was calculated. The Spearman rank-order correla- tion coefficient for this case was -0.34 , indicative of a weak correlation between the $\mathrm{K}_{\mathrm{app}}$ and $\mathrm{D}_{\text {app }}$. Fig $3 \mathrm{D}$ displays the $\mathrm{D}_{\text {app }}$ versus $\mathrm{K}_{\text {app }}$ plot of the primary tumor of patient 3 (Fig $3 B)$. In this case, the Spearman rank-order correlation is -0.90 . The range of absolute Spearman rho $(|\rho|)$ for all tumor lesions $(n=43)$ of the population was 0.1 to 0.95 , though for $44 \%$ of the lesions $(n=19)|\rho|<0.5$.

The heterogeneity values for the DWI-derived parameters, averaged over all tumor lesions $(n=43)$, were $\mathrm{hADC}_{\text {mono }}=$ $18.2 \pm 3.6, \mathrm{hD}_{\text {app }}=19.2 \pm 3.5$, and $\mathrm{hK}_{\mathrm{app}}=12.5 \pm 4.6$ (mean $\pm \mathrm{SD}$ ). The heterogeneity measure for the diffusion coefficient obtained by using the kurtosis analysis $\mathrm{hD}_{\text {app }}$ was statistically higher $(P<.05)$ than the value $\mathrm{hADC}_{\text {mono }}$ obtained by using the monoexponential analysis.

We identified 25 tumor lesions with necrosis and 18 lesions with no necrosis; specifically, 23 metastatic neck nodes and 2 primary tumors were necrotic, whereas 5 nodes and 14 primary tumors were not necrotic. Due to the limited number of necrotic primary tumors $(n=2)$, no separate analysis was performed for the primary lesions. However, a separate analysis for the metastatic lymph nodes was performed, and it yielded significantly higher $\mathrm{ADC}_{\text {mono }}$ and $\mathrm{D}_{\text {app }}$ values and significantly lower $\mathrm{K}_{\mathrm{app}}$ values for the necrotic nodes $(P<.01)$. The differences in Spearman correlation coefficient between $\mathrm{K}_{\mathrm{app}}$ and $\mathrm{D}_{\mathrm{app}}$ and heterogeneity values $\mathrm{hADC}_{\text {mono }}, \mathrm{hD}_{\mathrm{app}}$, and $\mathrm{hK}_{\mathrm{app}}$ were not significant for the necrotic $(n=23)$ versus the non-necrotic $(n=5)$ neck nodal metastases $(P>.1)$.

\section{Discussion}

In this study, we demonstrated the feasibility of using diffusional non-Gaussian (kurtosis) modeling to fit DWI data acquired by using an extended b-value range in studies of phantoms and HN tumors. Diffusion kurtosis represents the extent to which the diffusion pattern of the water molecules deviates from a perfect Gaussian curve. ${ }^{8}$ Because the deviation from Gaussian behavior is governed by the complexity of the tissue in which the water is diffusing, the diffusional kurtosis can be regarded as an index of tissue microstructural complexity. ${ }^{8}$ The degree of diffusional non-Gaussianity may be clinically relevant, as it is thought to arise from diffusion barriers, such as cell membranes and organelles, and water compartments. ${ }^{8}$

There is substantial evidence that the diffusion decay is of non-monoexponential origin; however, the exact nature is not well understood. ${ }^{6}$ Studies have been done on brain and prostate cancer by using non-monoexponential analysis with either biexponential or stretched exponential or kurtosis analysis. ${ }^{5,6,8,28-30}$ In the present study we have used the approach of the diffusional kurtosis model, which does not provide information regarding intra- and extracellular compartments but allows an additional parameter, $\mathrm{K}_{\mathrm{app}}$, to be obtained that may potentially be more sensitive to pathologic changes. ${ }^{8}$ Other explanations for non-monoexponential diffusion behavior include macromolecule binding of water, ${ }^{31}$ restricted diffusion, ${ }^{32}$ and a regime of fast exchange between multiple compartments. ${ }^{33}$ The elucidation of the exact nature of the nonmonoexponential diffusion behavior and the theoretic validity of the kurtosis model were beyond the scope of the present study. A fair comparison of the kurtosis analysis with any other non-Gaussian analyses was beyond the scope of this study. A study by Minati et al compared 2 non-Gaussian mod- 

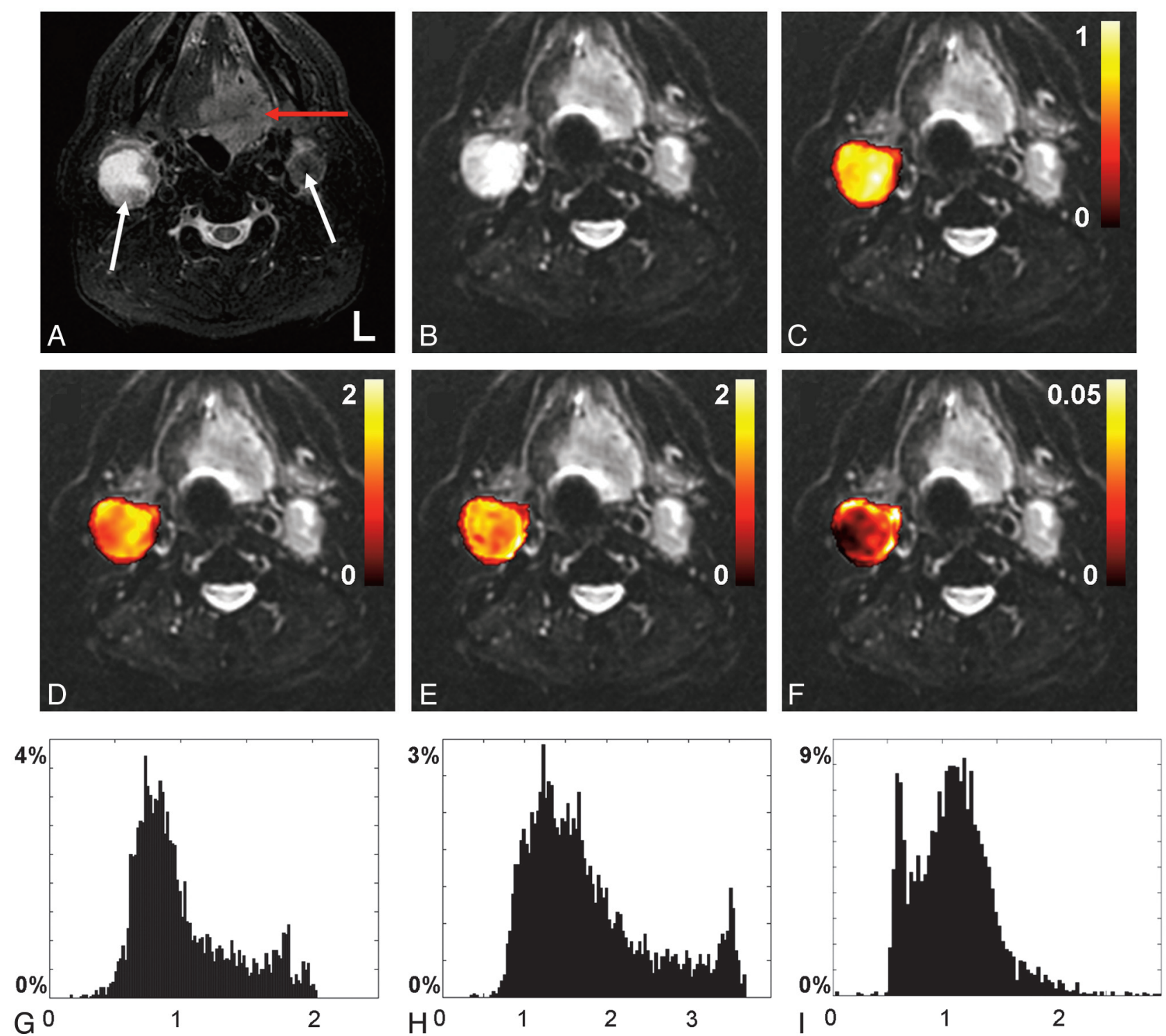

Fig 2. Axial MR images from the oral cavity of a male patient diagnosed with base of tongue cancer (patient 12). $A$, T2 short tau inversion recovery image. $B$, Realigned mean $b=0$ image. The red and white arrows in $A$ indicate the primary tumor and metastatic nodes, respectively. $C-F$ display voxel-by-voxel calculation outcomes for the right node presented as mask overlays on the realigned mean $b=0$ image. $G-I$ display the corresponding histogram distribution plots of the measures from $C-E$. $C$ and $G$, Apparent diffusion coefficient obtained from monoexponential fitting $\left(10^{-3} \mathrm{~mm}^{2} / \mathrm{s}\right)$. $D$ and $H$, Apparent diffusion coefficient $\left(10^{-3} \mathrm{~mm}^{2} / \mathrm{s}\right),\left(E\right.$ and $\Lambda$ apparent kurtosis coefficient (dimensionless), and $(F)$ reduced $\chi^{2}$ error estimate, all obtained from the diffusional kurtosis analysis.

els (biexponential and kurtosis) for the analysis of brain DWI in healthy volunteers and concluded that based on their experimental data, it was not possible to favor 1 of the 2 models. ${ }^{34}$

As pointed out by Thoeny et $\mathrm{al}^{35}$ who assessed the influence of the choice of b-values on ADC values obtained from the parotid gland, it is essential to realize that the choice of b-values has a great impact on the magnitude of the ADC values, especially when calculated by using a monoexponential approach. DWI by using low b-values generally yields higher ADC values than does DWI by using high b-values. This difference can be attributed to the non-monoexponential behavior of the DWI signal intensity, which points toward the potential benefits of using non-monoexponential fitting (eg, biexponential or non-Gaussian fitting). If one chooses to perform a non-Gaussian fitting approach on DWI data obtained over an extended b-value range, it is important to consider the range of b-values. First, it is essential to include relatively low b-values $\left(50 \mathrm{~s} / \mathrm{mm}^{2}\right)$, as some tissues display rapid signal intensity attenuation with low b-values, followed by a more gradual signal intensity reduction. ${ }^{1}$ This initial rapid reduction is thought to be due to vascular capillary perfusion. ${ }^{1} \mathrm{Sec}-$ ond, it is advisable to include high b-values $\left(>1000 \mathrm{~s} / \mathrm{mm}^{2}\right)$, as this is better for modeling the non-monoexponential nature of the decaying signal intensities. The anatomy of the head and neck region, with the intimate presence of air, bone, and acute changes in tissue bulk, promotes unwanted regional field distortions. ${ }^{36}$ Therefore, high b-values up to $3000 \mathrm{~s} / \mathrm{mm}^{2}$, typically used in brain ${ }^{37}$ and prostate, ${ }^{30}$ will not be achievable in head and neck due to the poorer SNR obtained with the current MR imaging acquisition technique. In the present study, the diffusion-weighted images acquired with $b=1500 \mathrm{~s} / \mathrm{mm}^{2}$ are the images with the lowest SNR. These images still display excellent SNR characteristics (median, 42; range, 21-87). Additionally, the fits of the diffusion-weighted images in the 

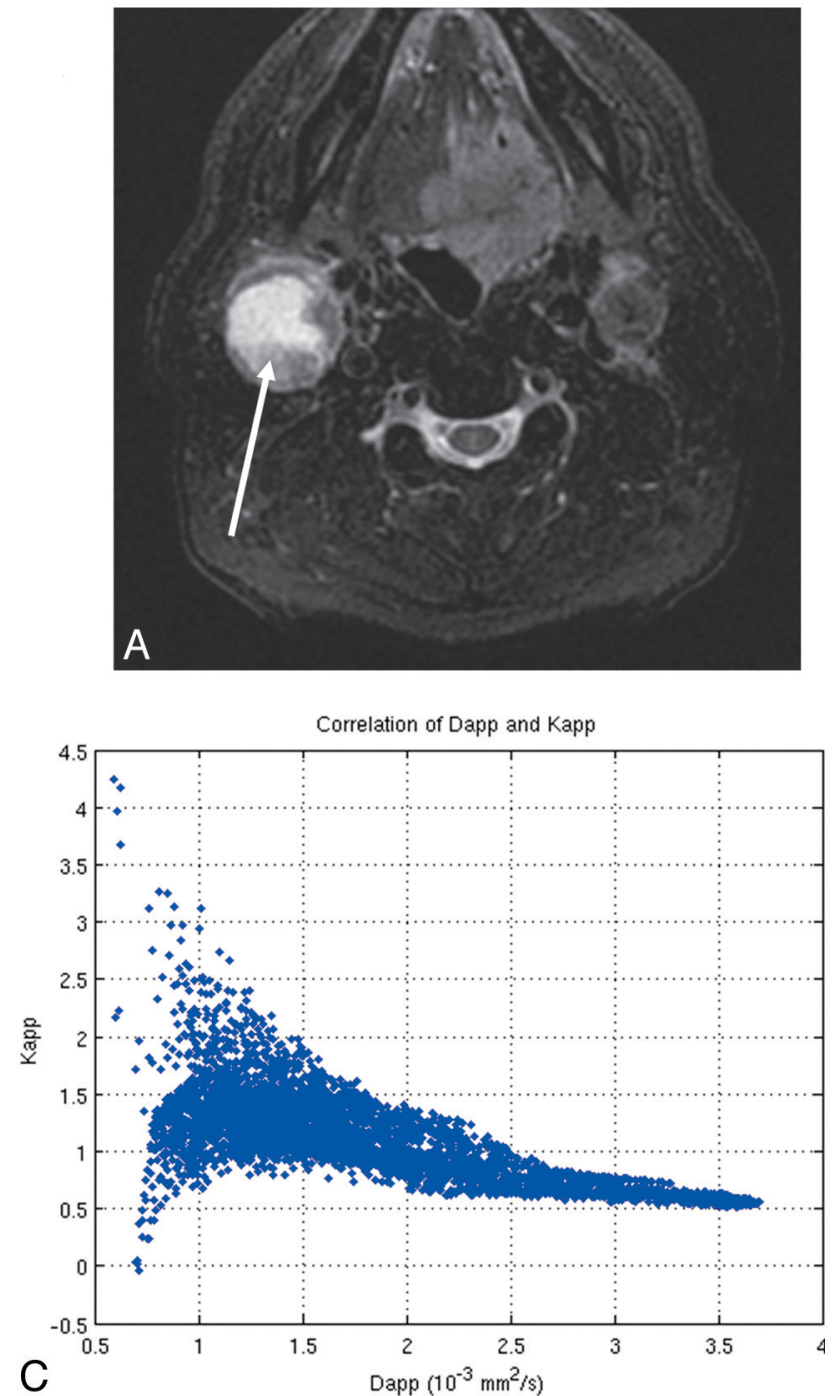
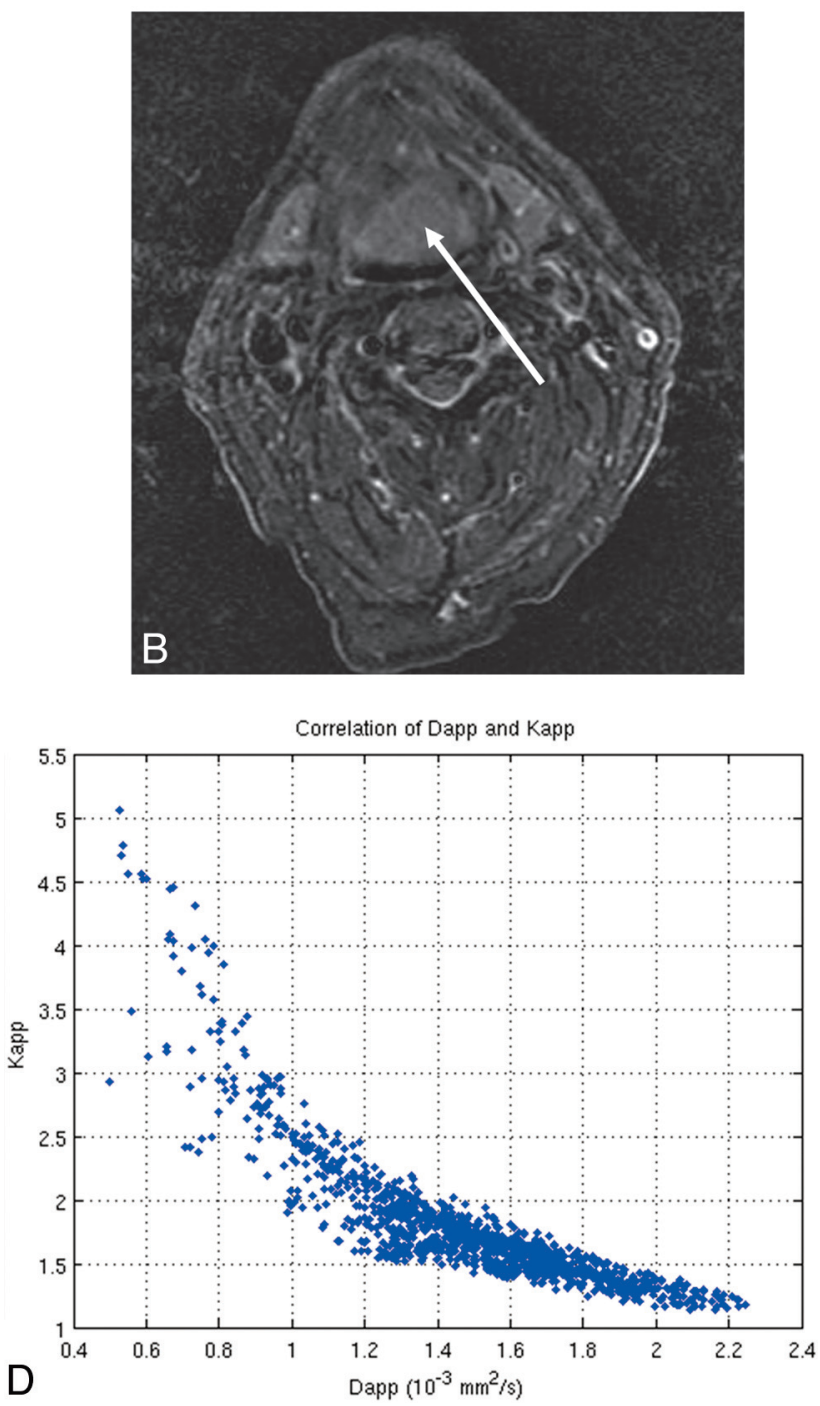

Fig 3. T2 short tau inversion recovery images for $(A)$ the right neck metastatic lymph node of patient 12 and $(B)$ primary base of tongue tumor of patient $3($ ROls are indicated with a white arrow). $C$ and $D$ display the corresponding scatterplots showing the correlation between the $\mathrm{D}_{\text {app }}$ (average apparent diffusion coefficient) and the $K_{\text {app }}$ (apparent diffusional kurtosis) obtained from the diffusional kurtosis analysis. The Spearman rank-order correlation coefficient is -0.34 for $C$, indicating a weak correlation, and -0.90 for $D$, indicating a strong negative correlation.

present study display beneficial reduced $\chi^{2}$ goodness-of-fit characteristics (reduced $\chi^{2}<0.15$ ). Therefore, we are confident about the quality of our data and in particular our choice of a maximum b-value of $1500 \mathrm{~s} / \mathrm{mm}^{2}$.

In the present study the average $\mathrm{ADC}_{\text {mono }}$ values for various structures in the 17 patients with HNSCC (Table 2) are in agreement with previously reported values in the literature. ${ }^{18,38,39}$ We obtained an average $\mathrm{ADC}_{\text {mono }}$ value of $0.84 \pm$ $0.24 \times 10^{-3} \mathrm{~mm}^{2} / \mathrm{s}$ for metastatic HNSCC neck lymph nodes (Table 2), which is in close agreement with values previously reported by Kim et al of $0.77 \pm 0.11 \times 10^{-3} \mathrm{~mm}^{2} / \mathrm{s}^{38}$ and values reported by Vandecaveye et al of $0.85 \pm 0.27 \times 10^{-3}$ $\mathrm{mm}^{2} / \mathrm{s}^{39}$ Furthermore, the obtained $\mathrm{ADC}_{\text {mono }}$ for the spinal cord of $0.74 \pm 0.08 \times 10^{-3} \mathrm{~mm}^{2} / \mathrm{s}$ is in the range of the values described by Wang et al of $1.02 \pm 0.15 \times 10^{-3} \mathrm{~mm}^{2} / \mathrm{s}^{18}$

Note, however, that the average diffusion coefficients, the heterogeneity measures, and the necrosis assessment, as obtained in our study, should be interpreted with caution, due to the limited number of patients per structure (Table 2). However, our study is unique among DWI studies in HN cancers, as we performed a non-Gaussian analysis rather than the usual monoexponential analysis.

The ADC values estimated by using the monoexponential model were systematically lower than the $\mathrm{D}_{\text {app }}$ values obtained from the diffusional kurtosis model. This finding is in agreement with those of Lu et al, who observed the same phenomenon in brain tissue. ${ }^{3} \mathrm{Lu}$ et al attributed this discrepancy to the nature of the diffusional kurtosis model, which is essentially a second-order polynomial fitting model. This model will always give better fitting results than a linear model.

Our phantom experiments yielded a non- $0 \mathrm{~K}_{\text {app }}$ value for homogenized asparagus (which is known to have structure) and a $0 \mathrm{~K}_{\mathrm{app}}$ value for water (which does not have structure) as consistent with literature. ${ }^{8}$ Previous studies have shown the advantage of $K_{a p p}$ as a specific measure of tissue structure. ${ }^{3,8}$ Although $\mathrm{K}_{\mathrm{app}}$ was not necessarily independent of $\mathrm{D}_{\mathrm{app}}$ for all tumors, $44 \%$ of the tumors displayed a low correlation coefficient, suggesting that $\mathrm{K}_{\mathrm{app}}$ might provide additional information related to structure. Interestingly, $\mathrm{hD}_{\text {app }}$ was significantly higher than $\mathrm{hADC}_{\text {mono, }}$ which indicates that the kurtosis anal- 
ysis-derived diffusion coefficient $\mathrm{D}_{\mathrm{app}}$ is likely to be more sensitive to reflect heterogeneity than $\mathrm{ADC}_{\text {mono }}$. It was observed in a subanalysis of the neck nodal metastases that necrosis does not affect the correlation between $\mathrm{K}_{\mathrm{app}}$ and $\mathrm{D}_{\text {app }}$, as well as the heterogeneity measures $\mathrm{hADC}$ mono, $\mathrm{hD}_{\text {app }}$, and $\mathrm{hK}_{\text {app }}$. Our assessment of necrosis was limited, as we did not have pathology as a reference standard. However, we did observe significantly higher diffusion coefficients for necrotic metastatic lymph nodes than for non-necrotic nodes, which indicates that our necrosis assessment was accurate. As reflected in our small population study, necrosis may not have contributed significantly to the additional information retrieved by using the kurtosis analysis. Other processes such as hypoxia might play a role, ${ }^{40}$ yet our current study design did not incorporate hypoxia measurements. Future studies with pathology might provide better insight. Although the exact nature of $\mathrm{K}_{\mathrm{app}}$ has yet to be understood, both $\mathrm{D}_{\text {app }}$ and $\mathrm{K}_{\text {app }}$ may be more sensitive to pathologic changes in tumor physiology than diffusion coefficients obtained from monoexponential analysis.

In future studies, the parameters $\mathrm{D}_{\mathrm{app}}$ and $\mathrm{K}_{\mathrm{app}}$ provided by the kurtosis model could offer potential value for longitudinal studies assessing early response in $\mathrm{HN}$ cancer patients undergoing chemoradiation therapy.

Recently, there has been a shift to imaging at higher magnetic field strengths (3T or higher), as this provides a higher SNR, resulting in better spatial resolution. ${ }^{36}$ However, imaging at higher magnetic field strengths is not without challenges, especially in the head and neck, where images can be degraded due to susceptibility artifacts. ${ }^{36}$ Srinivasan et al have reported initial studies at $3 \mathrm{~T}$ similar to the ones at $1.5 \mathrm{~T}$ showing that mean ADC values were able to differentiate between benign and malignant pathology in the head and neck regions. ${ }^{16,41}$

In addition to obtaining multiple diffusion-weighted images over a range of different $b$-values, one can also acquire multiple diffusion-weighted images over an increased number of noncollinear directions (diffusion tensor imaging). ${ }^{42}$ This technique yields, in addition to the ADC, the fractional anisotropy, which provides quantitative information about the orientational coherence of cellular structures. Diffusion tensor imaging has been proved to provide information about architectural structural changes induced by cancer $^{42}$; however, head and neck structures do not necessarily reflect a fiber composition. Therefore, the fractional anisotropy measure will most likely not be affected drastically by neoplastic head and neck tissue changes.

The study has few limitations. First, it is a cross-sectional feasibility study that analyzed data from only 17 patients to assess the benefits of the non-Gaussian kurtosis analysis of DW images. Second, although we were able to show that the kurtosis model yields a significantly better fit than does standard monoexponential fitting, we were not able to assess the pathologic relevance and sensitivity of the calculated diffusion coefficients. Third, although the MR imaging examinations were performed at least 1 week after biopsy, there is still a possibility that these biopsies might have influenced the DWIderived parameters. However, visual inspection did not reveal any postbiopsy changes. Finally, the exact nature of the nonGaussian behavior still needs to be elucidated.

\section{Conclusions}

The use of a diffusional non-Gaussian (kurtosis) model to analyze DWI data acquired by using an extended b-value range in HNSCC is feasible. The diffusional non-Gaussian model yields a significantly better fit of the experimental data than does monoexponential fitting. Furthermore, it provides an additional parameter, $K_{a p p}$, potentially with added value.

\section{Acknowledgments}

The authors are grateful to MR imaging technologists Greg Nyman and colleagues for their great efforts to perform all MR imaging examinations. We also thank Dr Yousef Mazaheri for stimulating discussions.

\section{References}

1. Koh DM, Collins DJ. Diffusion-weighted MRI in the body: applications and challenges in oncology. AJR Am J Roentgenol 2007;188:1622-35

2. Basser PJ, Jones DK. Diffusion-tensor MRI: theory, experimental design and data analysis - a technical review. NMR Biomed 2002;15:456-67

3. Lu H, Jensen JH, Ramani A, et al. Three-dimensional characterization of nongaussian water diffusion in humans using diffusion kurtosis imaging. NMR Biomed 2006;19:236-47

4. Karger J. NMR self-diffusion studies in heterogeneous systems. Adv Colloid Interface Sci 1985;23:129-48

5. Niendorf T, Norris DG, Leibfritz D. Detection of apparent restricted diffusion in healthy rat brain at short diffusion times. Magn Reson Med 1994;32:672-77

6. Mulkern RV, Gudbjartsson H, Westin CF, et al. Multi-component apparent diffusion coefficients in human brain. NMR Biomed 1999;12:51-62

7. Bennett KM, Schmainda KM, Bennett RT, et al. Characterization of continuously distributed cortical water diffusion rates with a stretched-exponential model. Magn Reson Med 2003;50:727-34

8. Jensen JH, Helpern JA, Ramani A, et al. Diffusional kurtosis imaging: the quantification of non-Gaussian water diffusion by means of magnetic resonance imaging. Magn Reson Med 2005;53:1432-40

9. Abdel Razek AA, Gaballa G, Elhawarey G, et al. Characterization of pediatric head and neck masses with diffusion-weighted MR imaging. Eur Radiol 2009;19:201-08

10. Sumi M, Ichikawa Y, Nakamura T. Diagnostic ability of apparent diffusion coefficients for lymphomas and carcinomas in the pharynx. Eur Radiol 2007;17:2631-37

11. Sumi M, Nakamura T. Diagnostic importance of focal defects in the apparent diffusion coefficient-based differentiation between lymphoma and squamous cell carcinoma nodes in the neck. Eur Radiol 2009;19:975-81

12. Abdel Razek AA, Megahed AS, Denewer A, et al. Role of diffusion-weighted magnetic resonance imaging in differentiation between the viable and necrotic parts of head and neck tumors. Acta Radiol 2008;49:364-70

13. Abdel Razek AA, Sadek AG, Kombar OR, et al. Role of apparent diffusion coefficient values in differentiation between malignant and benign solitary thyroid nodules. AJNR Am J Neuroradiol 2008;29:563-68

14. Maeda M, Kato H, Sakuma H, et al. Usefulness of the apparent diffusion coefficient in line scan diffusion-weighted imaging for distinguishing between squamous cell carcinomas and malignant lymphomas of the head and neck. AJNR Am J Neuroradiol 2005;26:1186-92

15. Matsushima N, Maeda M, Takamura M, et al. Apparent diffusion coefficients of benign and malignant salivary gland tumors: comparison to histopathological findings. J Neuroradiol 2007;34:183-89

16. Srinivasan A, Dvorak R, Perni K, et al. Differentiation of benign and malignant pathology in the head and neck using $3 \mathrm{~T}$ apparent diffusion coefficient values: early experience. AJNR Am J Neuroradiol 2008;29:40-44

17. Sumi M, Sakihama N, Sumi T, et al. Discrimination of metastatic cervical lymph nodes with diffusion-weighted MR imaging in patients with head and neck cancer. AJNR Am J Neuroradiol 2003;24:1627-34

18. Wang J, Takashima S, Takayama F, et al. Head and neck lesions: characterization with diffusion-weighted echo-planar MR imaging. Radiology 2001;220: 621-30

19. Yerli H, Agildere AM, Aydin E, et al. Value of apparent diffusion coefficient calculation in the differential diagnosis of parotid gland tumors. Acta Radiol 2007;48:980-87

20. Dirix P, De Keyzer F, Vandecaveye V, et al. Diffusion-weighted magnetic resonance imaging to evaluate major salivary gland function before and after radiotherapy. Int J Radiat Oncol Biol Phys 2008;71:1365-71

21. Kato H, Kanematsu M, Tanaka O, et al. Head and neck squamous cell carcinoma: usefulness of diffusion-weighted MR imaging in the prediction of a neoadjuvant therapeutic effect. Eur Radiol 2009;19:103-09

22. Vandecaveye V, De Keyzer F, Nuyts S, et al. Detection of head and neck squa- 
mous cell carcinoma with diffusion weighted MRI after (chemo)radiotherapy: correlation between radiologic and histopathologic findings. Int J Radiat Oncol Biol Phys 2007;67:960-71

23. Abdel Razek AA, Kandeel AY, Soliman N, et al. Role of diffusion-weighted echo-planar MR imaging in differentiation of residual or recurrent head and neck tumors and posttreatment changes. AJNR Am J Neuroradiol 2007;28: $1146-52$

24. Vandecaveye V, de Keyzer F, Vander Poorten V, et al. Evaluation of the larynx for tumour recurrence by diffusion-weighted MRI after radiotherapy: initial experience in four cases. Br J Radiol 2006;79:681-87

25. Koc O, Paksoy Y, Erayman I, et al. Role of diffusion weighted MR in the discrimination diagnosis of the cystic and/or necrotic head and neck lesions. Eur J Radiol 2007;62:205-13

26. Rorden C, Brett M. Stereotaxic display of brain lesions. Behav Neurol 2000;12:191-200

27. Lee $\mathrm{CH}$, Choi JW, Kim KA, et al. Usefulness of standard deviation on the histogram of ultrasound as a quantitative value for hepatic parenchymal echo texture; preliminary study. Ultrasound Med Biol 2006;32:1817-26

28. Mulkern RV, Barnes AS, Haker SJ, et al. Biexponential characterization of prostate tissue water diffusion decay curves over an extended $b$-factor range. Magn Reson Imaging 2006;24:563-68

29. Bennett KM, Hyde JS, Rand SD, et al. Intravoxel distribution of DWI decay rates reveals C6 glioma invasion in rat brain. Magn Reson Med 2004;52:9941004

30. Shinmoto $\mathrm{H}$, Oshio $\mathrm{K}$, Tanimoto $\mathrm{A}$, et al. Biexponential apparent diffusion coefficients in prostate cancer. Magn Reson Imaging 2000;27:355-59

31. Lyng $\mathrm{H}$, Haraldseth $\mathrm{O}$, Rofstad EK. Measurement of cell density and necrotic fraction in human melanoma xenografts by diffusion weighted magnetic resonance imaging. Magn Reson Med 2000;43:828-36
32. Sukstanskii AL, Yablonskiy DA. Effects of restricted diffusion on MR signal formation. J Magn Reson 2002;157:92-105

33. Kiselev VG, Il'yasov KA. Is the "biexponential diffusion" biexponential? Magn Reson Med 2007;57:464-69

34. Minati L, Aquino D, Rampoldi S, et al. Biexponential and diffusional kurtosis imaging, and generalised diffusion-tensor imaging (GDTI) with rank-4 tensors: a study in a group of healthy subjects. MAGMA 2007;20:241-53

35. Thoeny HC, De Keyzer F, Boesch C, et al. Diffusion-weighted imaging of the parotid gland: influence of the choice of $b$-values on the apparent diffusion coefficient value. J Magn Reson Imaging 2004;20:786-90

36. Aygun N, Zinreich SJ. Head and neck imaging at 3T. Magn Reson Imaging Clin N Am 2006;14:89-95

37. Dudink J, Larkman DJ, Kapellou O, et al. High b-value diffusion tensor imaging of the neonatal brain at 3T. AJNR Am J Neuroradiol 2008;29:1966-72

38. Kim JK, Kim KA, Park BW, et al. Feasibility of diffusion-weighted imaging in the differentiation of metastatic from nonmetastatic lymph nodes: early experience. J Magn Reson Imaging 2008;28:714-19

39. Vandecaveye V, De Keyzer F, Vander Poorten V, et al. Head and neck squamous cell carcinoma: value of diffusion-weighted MR imaging for nodal staging. Radiology 2009;251:134-46

40. Jansen JF, Schoder H, Lee NY, et al. Noninvasive assessment of tumor microenvironment using dynamic contrast enhanced MRI and 18F-fluoromisonidazole PET imaging in neck nodal metastases. Int J Radiat Oncol Biol Phys 2009 Nov 9 [Epub ahead of print]

41. Srinivasan A, Dvorak R, Rohrer S, et al. Initial experience of 3-Tesla apparent diffusion coefficient values in characterizing squamous cell carcinomas of the head and neck. Acta Radiol 2008;49:1079-84

42. Manenti G, Carlani M, Mancino S, et al. Diffusion tensor magnetic resonance imaging of prostate cancer. Invest Radiol 2007;42:412-19 